\title{
smwrGraphs-An R Package for Graphing Hydrologic Data, Version 1.1.2
}

Open-File Report 2016-1188

Supersedes USGS Open-File Report 2015-1202 



\section{smwrGraphs-An R Package for Graphing Hydrologic Data, Version 1.1.2}

By David L. Lorenz and Aliesha L. Diekoff

Open-File Report 2016-1188

Supersedes USGS Open-File Report 2015-1202

U.S. Department of the Interior

U.S. Geological Survey 


\section{U.S. Department of the Interior SALLY JEWELL, Secretary}

\section{U.S. Geological Survey Suzette M. Kimball, Director}

\section{U.S. Geological Survey, Reston, Virginia: 2017}

For more information on the USGS - the Federal source for science about the Earth, its natural and living resources, natural hazards, and the environment-visit https://www.usgs.gov or call 1-888-ASK-USGS.

For an overview of USGS information products, including maps, imagery, and publications, visit https://www.usgs.gov/pubprod/.

Any use of trade, firm, or product names is for descriptive purposes only and does not imply endorsement by the U.S. Government.

Although this information product, for the most part, is in the public domain, it also may contain copyrighted materials as noted in the text. Permission to reproduce copyrighted items must be secured from the copyright owner.

Suggested citation:

Lorenz, D.L., and Diekoff, A.L., 2017, smwrGraphs-An R package for graphing hydrologic data, version 1.1.2:

U.S. Geological Survey Open-File Report 2016-1188, 17 p., https://doi.org/10.3133/ofr20161188. [Supersedes USGS Open-File Report 2015-1202.]

ISSN 2331-1258 (online) 


\section{Contents}

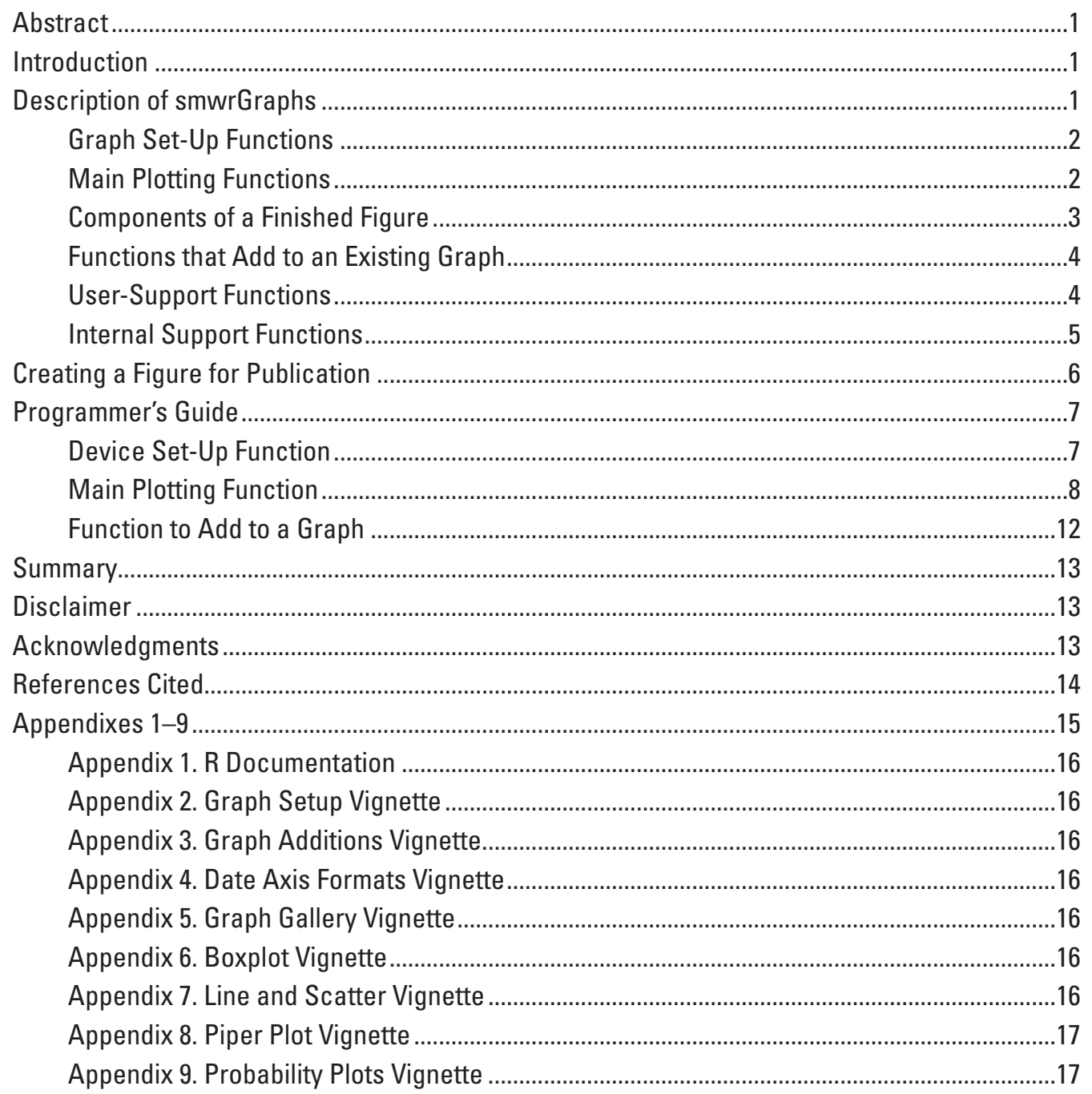

\section{Figures}

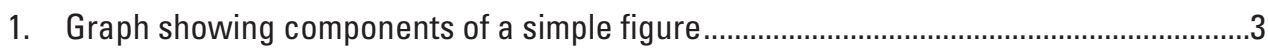

2. R code for a specific example of a combination grap.....................................................6

\section{Tables}

1. Functions in the smwrGraphs package that set up the graphics environment .................2

2. Main plotting functions in the smwrGraphs package ....................................................2

3. Functions in the smwrGraphs package that add information or a plot to an existing graph.

4. User-support functions in the smwrGraphs package .....................................................

5. Internal support functions in the smwrGraphs package ................................................ 



\title{
smwrGraphs-An R Package for Graphing Hydrologic Data, Version 1.1.2
}

\author{
By David L. Lorenz and Aliesha L. Diekoff
}

\begin{abstract}
This report describes an R package called smwrGraphs, which consists of a collection of graphing functions for hydrologic data within $\mathrm{R}$, a programming language and software environment for statistical computing. The functions in the package have been developed by the U.S. Geological Survey to create high-quality graphs for publication or presentation of hydrologic data that meet U.S. Geological Survey graphics guidelines.
\end{abstract}

\section{Introduction}

The U.S. Geological Survey (USGS) uses graphics to present and describe hydrologic data in publications, presentations, and other venues. In the book "Statistical Methods in Water Resources," Helsel and Hirsch (2002) discuss the importance of graphics for analyzing and describing hydrologic data. The purpose of this report is to describe a USGS created R package called smwrGraphs, which consists of a collection of graphing functions for hydrologic data within $\mathrm{R}$, a programming language and software environment for statistical computing. The functions in the package have been developed by the USGS to create high-quality graphs for publication or presentation of hydrologic data that meet USGS graphics guidelines.

It is assumed that the reader of this report has a rudimentary knowledge of the R language and environment. General information on $\mathrm{R}$ can be obtained from the R project at https:// www.r-project.org/ (particularly the Manuals page). Many books and online resources also provide information about using R.

\section{Description of smwrGraphs}

The R package smwrGraphs consists of a collection of functions to set up, create, and augment graphs within R. Functions in the package allow users to easily create simple, high-quality graphs and manage the data plotted to easily create descriptions of the plotted data. Additional functions are provided to add additional plots or annotation. Documentation for the functions is provided in appendix 1.

The functions in smwrGraphs are provided as a package in R (https://www.r-project.org/), an open source language and environment for statistical computing and graphics that runs on a variety of operating systems including UNIX ${ }^{\circledR}$, Linux $^{\circledR}$, Windows ${ }^{\circledR}$, and $\mathrm{Mac}^{\mathrm{OS}}{ }^{\circledR}$. R can be extended for additional functionality using packages. Additional information on the installation and administration of $\mathrm{R}$ and packages that extend $\mathrm{R}$ is available in the manual " $\mathrm{R}$ Installation and Administration" (R Development Core Team, 2011).

Many of the functions in the smwrGraphs package, version 1.1.0, have been ported from the USGS library for $\mathrm{S}+$, a commercial statistical and graphical analysis software package (Lorenz and others, 2011). Other functions have been developed and used within the USGS since the publication of that report. All of the functions are in the public domain (see the "Disclaimer" section). In the text of this report, functions are given in bold italics, and arguments are given in italics.

The suggested citation for data from the smwrGraphs package can be acquired by using the citation function in R. The call is citation (package="smwrGraphs"). 


\section{Graph Set-Up Functions}

The graphics environment used by graphing functions in smwrGraphs must be set up by a call to the setPage function (for complete onscreen graphics device set up), setGD function (for a simple onscreen graphics device), setPDF function (for Portable Document Format [PDF] output), setPNG function (for Portable Network Graphics [PNG] output), setSweave function (within a SWeave script), or setKnitr function (within a knitr script). A call to one of those set-up functions can be followed by a call to the setLayout function to set up the layout for one or more graphs on the device. A call to the setGraph function is required to set up a specific graph area if setLayout is used. A complete list of graph set-up functions is provided in table 1 .

Table 1. Functions in the smwrGraphs package that set up the graphics environment.

[PDF, Portable Document Format; PNG, Portable Network Graphics]

\begin{tabular}{ll}
\hline $\begin{array}{c}\text { Function } \\
\text { name }\end{array}$ & \multicolumn{1}{c}{ Description } \\
\hline setGD & Set up an onscreen graphics device. \\
setGraph & Set up the graph area for a single graph. \\
setKnitr & $\begin{array}{l}\text { Set up a PDF file as the graphics device within a knitr } \\
\text { script. }\end{array}$ \\
setLayout & Set up the layout for one or more graphs and optional \\
& explanation. \\
setPage & Set up an onscreen graphics device. \\
setPDF & Set up a PDF file as the graphics device. \\
setPNG & Set up a PNG file as the graphics device. \\
setSplom & Set up the layout for a scatter plot matrix. \\
setSweave & Set up a PDF file as the graphics device within a \\
& SWeave script. \\
preSurface & Set up a surface graph. \\
\hline
\end{tabular}

\section{Main Plotting Functions}

The main plotting functions create graphs of data. All functions have options for controlling what is plotted and for controlling the axes. Table 2 is a list of these functions.

Table 2. Main plotting functions in the smwrGraphs package.

\begin{tabular}{|c|c|}
\hline $\begin{array}{c}\text { Function } \\
\text { name }\end{array}$ & Description \\
\hline areaPlot & Graph that highlights the area between curves. \\
\hline biPlot & Graph two pairs of data. \\
\hline boxPlot & Create a boxplot. \\
\hline colorPlot & $\begin{array}{l}\text { Create a scatter plot where the color of each symbol } \\
\text { is set by another variable. }\end{array}$ \\
\hline condition & $\begin{array}{l}\text { Facilitate producing a series of graphs conditioned } \\
\text { by a grouping variable. }\end{array}$ \\
\hline contourPlot & Contour the data. \\
\hline corGram & Create a correlogram from irregularly spaced data. \\
\hline dotPlot & $\begin{array}{l}\text { Create a scatter plot where the } y \text {-axis is a categori- } \\
\text { cal variable. }\end{array}$ \\
\hline ecdfPlot & $\begin{array}{l}\text { Create a graph of empirical distribution function of } \\
\text { data. }\end{array}$ \\
\hline histGram & Create a histogram. \\
\hline piperPlot & Create Piper diagram. \\
\hline probPlot & Create a probability plot. \\
\hline qqPlot & Create a Q-Q plot or Q-normal plot. \\
\hline reportGraph & Create a report of any $\mathrm{R}$ object in a graph. \\
\hline scalePlot & $\begin{array}{l}\text { Create a graph with a fixed relation between the } \\
x-\text { and y-axes. }\end{array}$ \\
\hline seasonPlot & $\begin{array}{l}\text { Graph time-series data over a year rather than by } \\
\text { time. }\end{array}$ \\
\hline seriesPlot & Graph seasonal data by season. \\
\hline splomPlot & Create a scatter plot matrix. \\
\hline surfacePlot & Create a surface graph. \\
\hline stiffPlot & Create a Stiff diagram. \\
\hline ternaryPlot & $\begin{array}{l}\text { Create a ternary plot, also called trilinear or trian- } \\
\text { gular plot. }\end{array}$ \\
\hline timePlot & Graph time-series data by time. \\
\hline transPlot & Create a scatter plot using arbitrary axes transforms. \\
\hline xyPlot & Create a line/scatter plot. \\
\hline
\end{tabular}




\section{Components of a Finished Figure}

The finished figure can contain a single graph or multiple graphs. The example in figure 1 identifies the components of a figure showing a single graph. The arguments in the main plotting functions that affect the drawing of each component or functions that add to a graph are described in the following paragraphs.

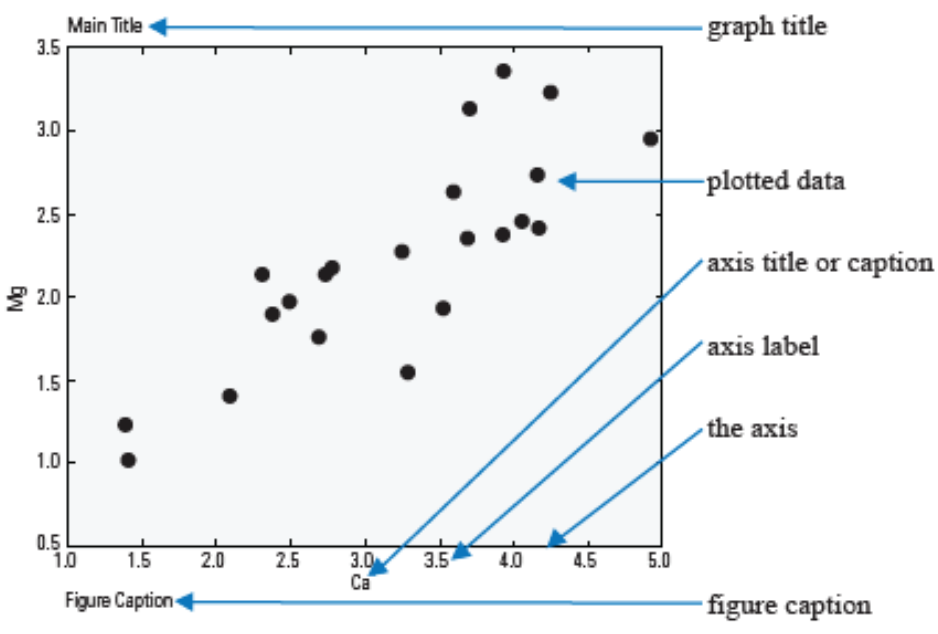

Figure 1. Components of a simple figure.

The graph area in figure 1 contains the components of a simple graph, including the figure caption. The graph area is defined using the graph set-up functions, such as setPDF and setLayout.

The plotted data are defined by the $x, y$, and Plot arguments. The $x$ and $y$ arguments define the data to be plotted, and Plot defines how they are to be plotted, as points or lines, the color, and so forth.

The y-axis is defined by the yaxis.log, yaxis.rev, and yaxis.range arguments, with similar names for the $\mathrm{x}$-axis. Not all main plotting functions support all of those arguments. The yaxis.log and yaxis.rev arguments require logical values: TRUE sets up the axis in log-scale or decreasing scale (reversed), respectively, whereas FALSE sets up a linear axis and increasing scale, respectively. The yaxis.range argument defines the exact range for the axis, which can be used to set the same range for multiple graphs or fine tune the automatic computation of the range. The default, $c$ (NA, NA), computes the range of the data for each axis, extends the range by a small amount to prevent plotting points on the axis, and then computes "pretty" limits (chosen so that they are 1, 2, or 5 times a power of 10). The actual axis length is controlled by the size of the graph area and the margin argument. The default value for margin is $c(N A, N A, N A, N A)$, which maximizes axis length while accounting for necessary axis labels and titles. In general, the default margin value should not need to be changed, except when the setLayout function is called to set up shared axes, or when right axis labels are needed.

The axis labels are defined by the ylabels and xlabels arguments for the y-axis and x-axis, respectively. In general, the default value is "Auto," which inserts a reasonable number of labels given the range of the axis. An integer value also can be supplied, which inserts that number of labels for the axis, but the number of labels inserted is only a guide and may need adjustment to get the actual desired result. The ylabels or xlabels argument also can be a list that can help set up the axis and labels; if a list, then the component names must match the arguments for the setAxis function.

The y-axis or $\mathrm{x}$-axis title, referred to as the axis caption in the USGS publications guide for illustrations (U.S. Geological Survey, written commun., October 2015), is defined by the ytitle or xtitle argument, respectively. The axis title is a single character string. For most functions, it defaults to the name supplied to the $y$ and $x$ arguments.

The graph title, called the graph heading in the USGS publications guide for illustrations, is created by the addTitle function. The graph title is included here for completeness. In general, the graph title is used as a short identifier for the graph, rather than as a description of the graph. In many cases, it can be a single letter for a multipart figure.

The figure caption is a detailed description of the figure, not just a graph. The figure caption is defined by the caption argument in the main plotting functions but also can be added using the addCaption function. 


\section{Functions that Add to an Existing Graph}

Once a graph has been created, several functions can add features to that graph. These functions are listed in table 3 .

Table 3. Functions in the smwrGraphs package that add information or a plot to an existing graph.

\begin{tabular}{|c|c|}
\hline Function name & Description \\
\hline addAnnotation & Add text to a plot to annotate a feature. \\
\hline addArea & Add a shaded polygon to the graph. \\
\hline addAxisLabels & Label an axis. \\
\hline addBars & Add a bar chart to the graph. \\
\hline addCaption & Write a figure caption below the graph. \\
\hline addCI & $\begin{array}{l}\text { Add confidence interval lines to a simple linear } \\
\text { regression or Q-normal graph. }\end{array}$ \\
\hline addErrorBars & Add upper-lower error bars to data in a graph. \\
\hline addExplanation & $\begin{array}{l}\text { Create or add an explanation, also called key or } \\
\text { legend. }\end{array}$ \\
\hline addGrid & Add grid lines to a graph. \\
\hline addLabel & $\begin{array}{l}\text { Add text in the margin for specialized axis } \\
\text { labels. }\end{array}$ \\
\hline addMinorTicks & Add minor ticks to a graph. \\
\hline addPiper & Add to a Piper diagram. \\
\hline addSLR & $\begin{array}{l}\text { Add a simple linear regression line to a scatter } \\
\text { plot. }\end{array}$ \\
\hline addSmooth & Smooth data and add the line to the graph. \\
\hline addStiff & Add a Stiff diagram to a graph. \\
\hline addTable & Add a small table to a graph. \\
\hline addTernary & Add to a ternary plot. \\
\hline addTitle & Add a title above the graph. \\
\hline addXY & Add a line or scatter plot to a graph. \\
\hline labelPoints & Label individual points on a graph. \\
\hline refLine & $\begin{array}{l}\text { Add a horizontal, vertical, or simple linear } \\
\text { regression line to a graph. }\end{array}$ \\
\hline
\end{tabular}

\section{User-Support Functions}

The smwrGraphs package contains some functions that can assist the user in preparing graphs. User-support functions are listed in table 4. The functions ending in ".colors" (such as blueRed.colors) can be used to help the user define a set of colors for points, lines, or areas to distinguish different sets of data. The functions cov2Ellipse, dataEllipse, hull, interpLine, and paraSpline provide specialized data manipulation functions to assist the user in adding specialized plots to a graph.

Table 4. User-support functions in the smwrGraphs package.

\begin{tabular}{|c|c|}
\hline Function name & Description \\
\hline blueRed.colors & $\begin{array}{l}\text { Generate a set of colors ranging from blue to } \\
\text { red. }\end{array}$ \\
\hline coolWarm.colors & $\begin{array}{l}\text { Generate a set of colors ranging from cool to } \\
\text { warm. }\end{array}$ \\
\hline copyDemo & $\begin{array}{l}\text { Copy a demo script to the current or other } \\
\text { directory. }\end{array}$ \\
\hline cov2Ellipse & Construct an ellipse from a covariance matrix \\
\hline dataEllipse & $\begin{array}{l}\text { Construct an ellipse from } \mathrm{x} \text { - and } \mathrm{y} \text {-coordinate } \\
\text { data }\end{array}$ \\
\hline greenRed.colors & $\begin{array}{l}\text { Generate a set of colors ranging from green } \\
\text { to red. }\end{array}$ \\
\hline hull & Get the data points for a convex hull. \\
\hline interpLine & $\begin{array}{l}\text { Interpolate } x \text {-values from } y \text {-values or } y \text {-values } \\
\text { from } x \text {-values from plotted data. }\end{array}$ \\
\hline paraSpline & Interpolate data using a parametric spline. \\
\hline pastel.colors & Generate a range of colors in pastel shades. \\
\hline redBlue.colors & $\begin{array}{l}\text { Generate a set of colors ranging from red to } \\
\text { blue. }\end{array}$ \\
\hline redGreen.colors & $\begin{array}{l}\text { Generate a set of colors ranging from red to } \\
\text { green. }\end{array}$ \\
\hline strip.blanks & $\begin{array}{l}\text { Strip leading and trailing blanks from a char- } \\
\text { acter string. }\end{array}$ \\
\hline warmCool.colors & $\begin{array}{l}\text { Generate a set of colors ranging from warm } \\
\text { to cool. }\end{array}$ \\
\hline
\end{tabular}




\section{Internal Support Functions}

The smwrGraphs package has many internal support functions that are used within the main plotting functions and the other functions that add to a graph. These functions are listed in table 5 .

Table 5. Internal support functions in the smwrGraphs package.

\begin{tabular}{|c|c|}
\hline Function name & Description \\
\hline boxPlotStats & Compute the statistics for a boxplot. \\
\hline datePretty & $\begin{array}{l}\text { Prepare the ticks and labels for a date/ } \\
\text { time axis. }\end{array}$ \\
\hline frameWt, lineWt, stdWt & Set the line weight. \\
\hline getDist.fen & Get a distribution function for probPlot. \\
\hline linearPretty & $\begin{array}{l}\text { Prepare the ticks and labels for a linear } \\
\text { axis. }\end{array}$ \\
\hline logPretty & $\begin{array}{l}\text { Prepare the ticks and labels for a loga- } \\
\text { rithmic axis. }\end{array}$ \\
\hline namePretty & $\begin{array}{l}\text { Prepare the ticks and labels for a cat- } \\
\text { egorical axis. }\end{array}$ \\
\hline numericData & Convert any data to numeric values \\
\hline piperSubplot & $\begin{array}{l}\text { Create projected rhomboid plot in the } \\
\text { Piper plot. }\end{array}$ \\
\hline probPretty & $\begin{array}{l}\text { Prepare the ticks and labels for a prob- } \\
\text { ability axis. }\end{array}$ \\
\hline renderBoxPlot & Draw boxplots. \\
\hline renderBXP & The workhorse behind the boxplot. \\
\hline renderX, renderY & Draw axis ticks and labels. \\
\hline setAxis & Set up an axis from data. \\
\hline setColor & Convert anything to a valid color. \\
\hline setDefaults & Set defaults for plot control lists. \\
\hline setExplan & $\begin{array}{l}\text { Set up information for the explanation } \\
\text { based on the plot control lists. }\end{array}$ \\
\hline setGroupPlot & $\begin{array}{l}\text { Set up information when the symbology } \\
\text { is specified by group. }\end{array}$ \\
\hline setMargin & Set margin for a graph. \\
\hline setMultiPlot & $\begin{array}{l}\text { Set up information when the symbology } \\
\text { is specified for each point. }\end{array}$ \\
\hline setPlot & $\begin{array}{l}\text { Set the default value for a plot control } \\
\text { list }\end{array}$ \\
\hline ternarySubplot & Create ternary plot in the Piper plot. \\
\hline ticks.render & Draw ticks on an axis. \\
\hline timePretty & $\begin{array}{l}\text { Prepare the ticks and labels for a time- } \\
\text { series axis. }\end{array}$ \\
\hline transData & $\begin{array}{l}\text { Transform data to correspond to a spe- } \\
\text { cific axis type. }\end{array}$ \\
\hline transPretty & $\begin{array}{l}\text { Prepare the ticks and labels for an arbi- } \\
\text { trary transformed axis. }\end{array}$ \\
\hline
\end{tabular}

The smwrGraphs package has detailed help files for each function that may be accessed in the same manner as help for other R functions. Help features within R are further described in the manual "An Introduction to R" (Venables and others, 2011). The documentation for the smwrGraphs package is included in appendix 1 of this document. The smwrGraphs package also has vignettes (appendixes 2-9) that demonstrate the basic plotting capabilities and provide demo scripts that can be copied and can serve as starting scripts for more complicated graphs. 


\section{Creating a Figure for Publication}

The code in figure 2 provides an example of a combination graph that combines two or more forms into one graph. In the example, the general guidelines for setting the width of the figure in the call to the setPDF function is 3.5 for a single column figure and 7.167 for a double-column figure; the maximum height is 9.333, all of the units are in inches. However, if a full page figure is desired, the layout can be set to "portrait" or "landscape" depending on the orientation needed by the figure. Comments in the code in figure 2 and in other code included in this report are preceded by the \# symbol.

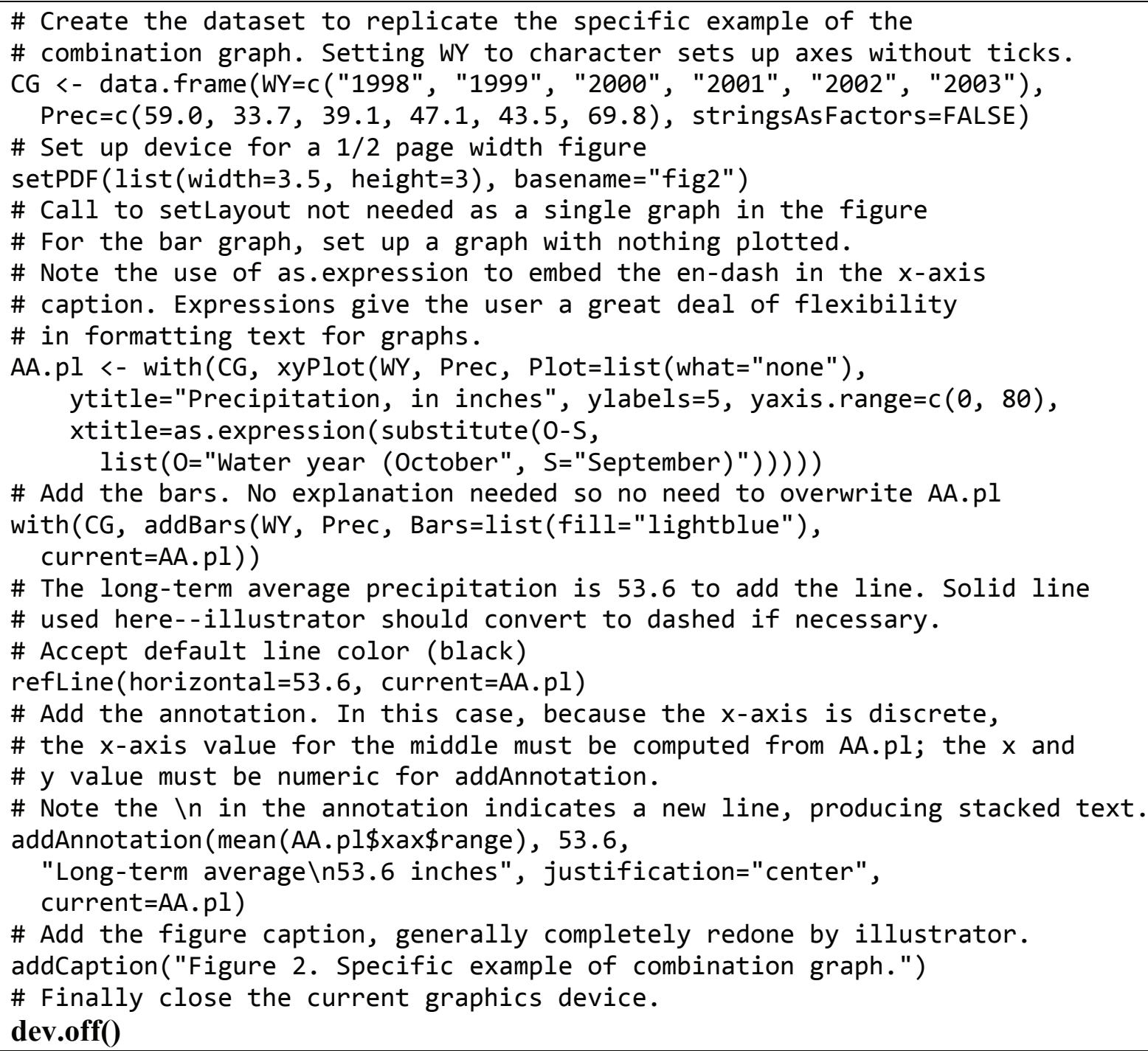

Figure 2. $\mathrm{R}$ code for a specific example of a combination graph. 


\section{Programmer's Guide}

This section of the report provides details about the structure of graphics set-up functions, main plotting functions, and functions that add to a graph. This section can be useful to anyone who wants to add a main plotting function or method to the package or develop a script that needs to call other plotting functions in base R. Many of the main plotting functions in smwrGraphs have methods for different types of arguments. The remainder of this section focuses on two functions that create or add to graphs, but the "HeatMap.r" demo script, found at https://github.com/USGS-R/smwrGraphs/tree/master/demo, also illustrates how to use low-level functions within the graphics environment in smwrGraphs.

\section{Device Set-Up Function}

This section of the report uses the setPDF function to describe the necessary steps for setting up a graphics device to work with the functions in smwrGraphs. The arguments to the function must represent those needed by the device; in general, the size of the device and the filename are needed. Code used for examples in this report is included as boxed code as follows for the setPDF function:

$$
\text { setPDF <-function(layout="portarit", basename"USGS", multiplefiles=FALSE) \{ }
$$

The options for the line weight factor, the pdf file output, and the font size must be set. The line weight factor (.lwt_factor) can generally be set to a specific value to control the line weights in the device, a value of 1 sets the correct values for line weights in a pdf file and can generally be used in any device. The options for pdf file output (.pdf_graph) must be set to TRUE to guarantee that specialized actions required to produce editable graphics are performed by the low-level functions. The font size must be set. In this case, the font size is set to a variable (fontSize) used later when pointsize is set to fontSize.

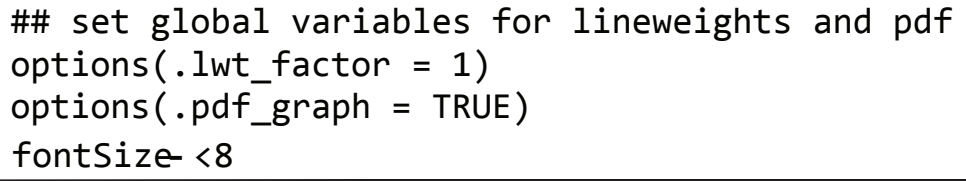

The following boxed code sets up the size of the graphics device and the figure size in inches. The variables set are used later in the actual call to set up the device and setting up the initial graphics parameters in the call to par. Because the focus of the output from the plotting functions in smwrGraphs is the production of graphs for publications, a fixed size is necessary. Note that the setRStudio function (see appendix 1) is anomalous because this function permits resizing of graphs; however, some graphs cannot be created using that output device.

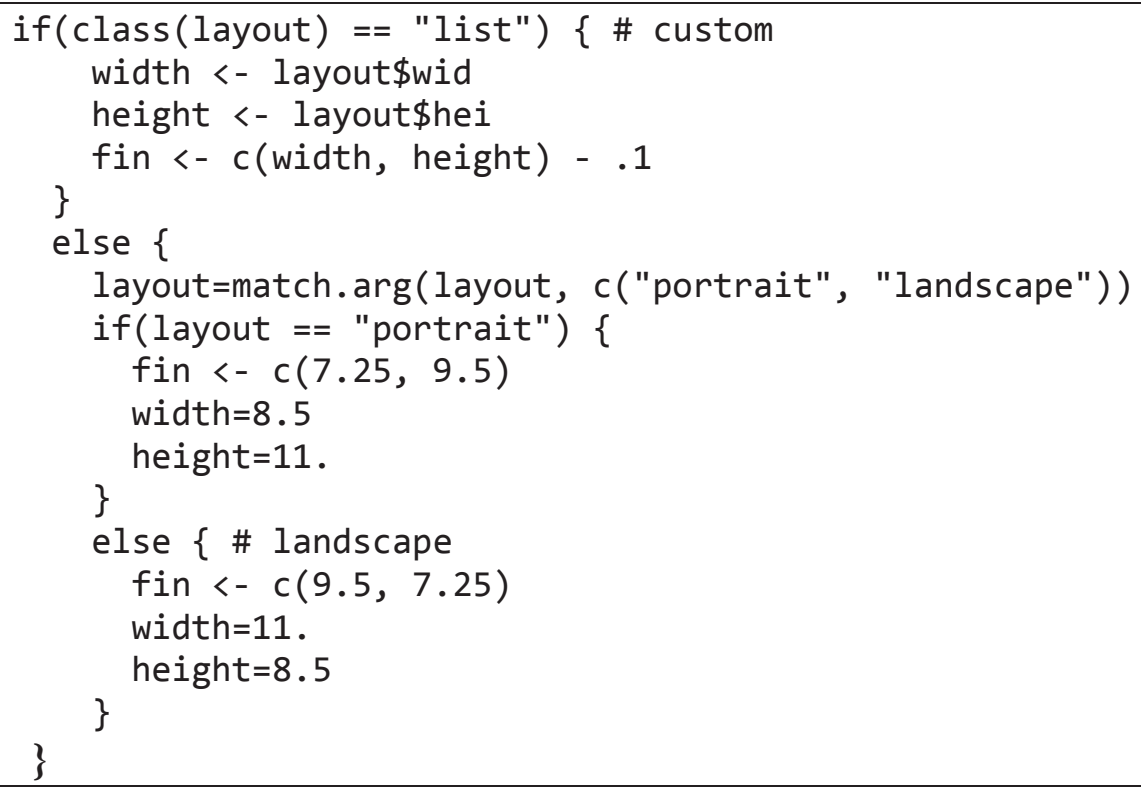


The output file must be set up and created; an example of this is shown in the following boxed code. In most cases, width, height, and the font size will be necessary options to select.

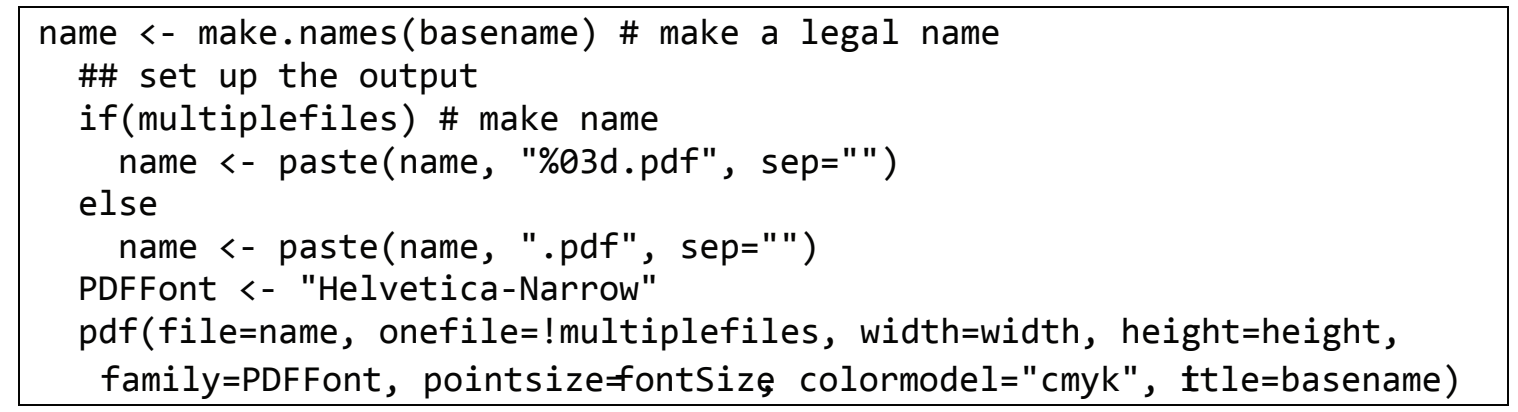

A font family called "USGS" must be set up for the device (as shown in the following boxed code) or warnings or errors will be generated from the main plotting functions and those functions that add any text. The actual function that registers the USGS font family will vary by device.

\#\# set up for export to PDF file.

if(all(names(pdfFonts()) != "USGS")) \# Check to see if already in the PDF font list pdfFonts("USGS" = Type1Font("Helvetica-Narrow", pdfFonts ("HelveticaNar row") [[1L]]\$metrics))

The final section of code sets up any necessary graphical parameters as shown in the following boxed code. The only required parameters are the figure size and the style of the axis labels (las, which should be set to 1). For invisible function, the device number and basename of the output file are returned invisibly in a tagged list. The device number could be used in the call to dev.off to close the output file, if multiple graphics devices are opened.

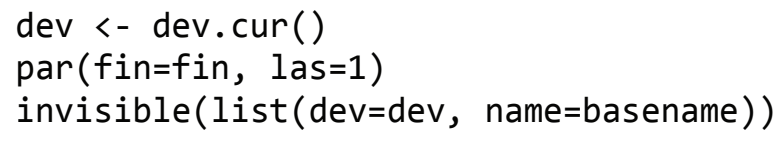

\section{Main Plotting Function}

This section of the report uses the areaPlot function to describe the main plotting function. Although the areaPlot function does not have methods for specific arguments, the extension to include different methods is straightforward and explained in many books and documentation for $\mathrm{R}$. The example code is presented below in boxed code with comments.

The formal arguments are set up in consistent order in the following boxed code for this example. The first set of arguments are the data to plot, in this case, $x$ and $y$. The next argument, Areas, in this example, describes how the data are to be plotted. The following set of arguments, beginning with yaxis or xaxis, set up the format of the axis. Not all axis format arguments may be appropriate for the graph; for example, yaxis.rev, which reverses the y-axis, is not supported for area plots. The remaining arguments set up the axis labels, axis titles (also called axis captions), and the figure caption. The last argument, margin, sets up specific margins, typically required only to force a specific size to the plot area. Additional plotting features, such as the reference or 1:1 line in the qqPlot function, typically follow the argument that describes how to plot the data.

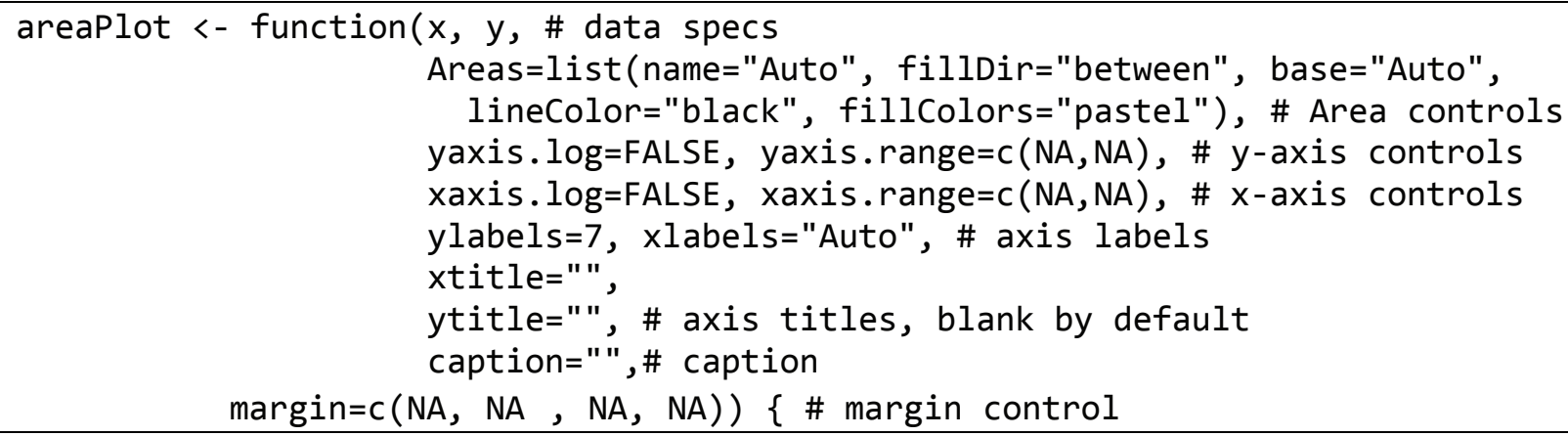


The following boxed code sets up the information needed to properly display the data. The setDefaults function is used for arguments other than Plot, which used the setPlot function. The use of setDefaults is required to fill in default options not specified by the user. The call to setGD is a convenience call to set up the correct graphics environment if one has not already been set up. The remaining code sets up the $\mathrm{x}$ - and $\mathrm{y}$-axis and the labeling. The functions in the smwrGraphs package that are used in this section include setAxis, which is typically called from do.call, and the functions to set up specific axis types, datePretty, linearPretty, logPretty, probPretty, timePretty, and transPretty (appendix 1). Other functions, like isCharLike, isDateLike, isGroupLike, or isNumberlike (in the smwrBase package (Lorenz, 2015) can be used to help determine how to set up the axis, but would not necessarily be used in method functions because the type of data would be known. The numericData function forces all data to be in a consistent numeric format; for example, data of class "Date" or "POSIXct" would be converted to comparable numeric values.

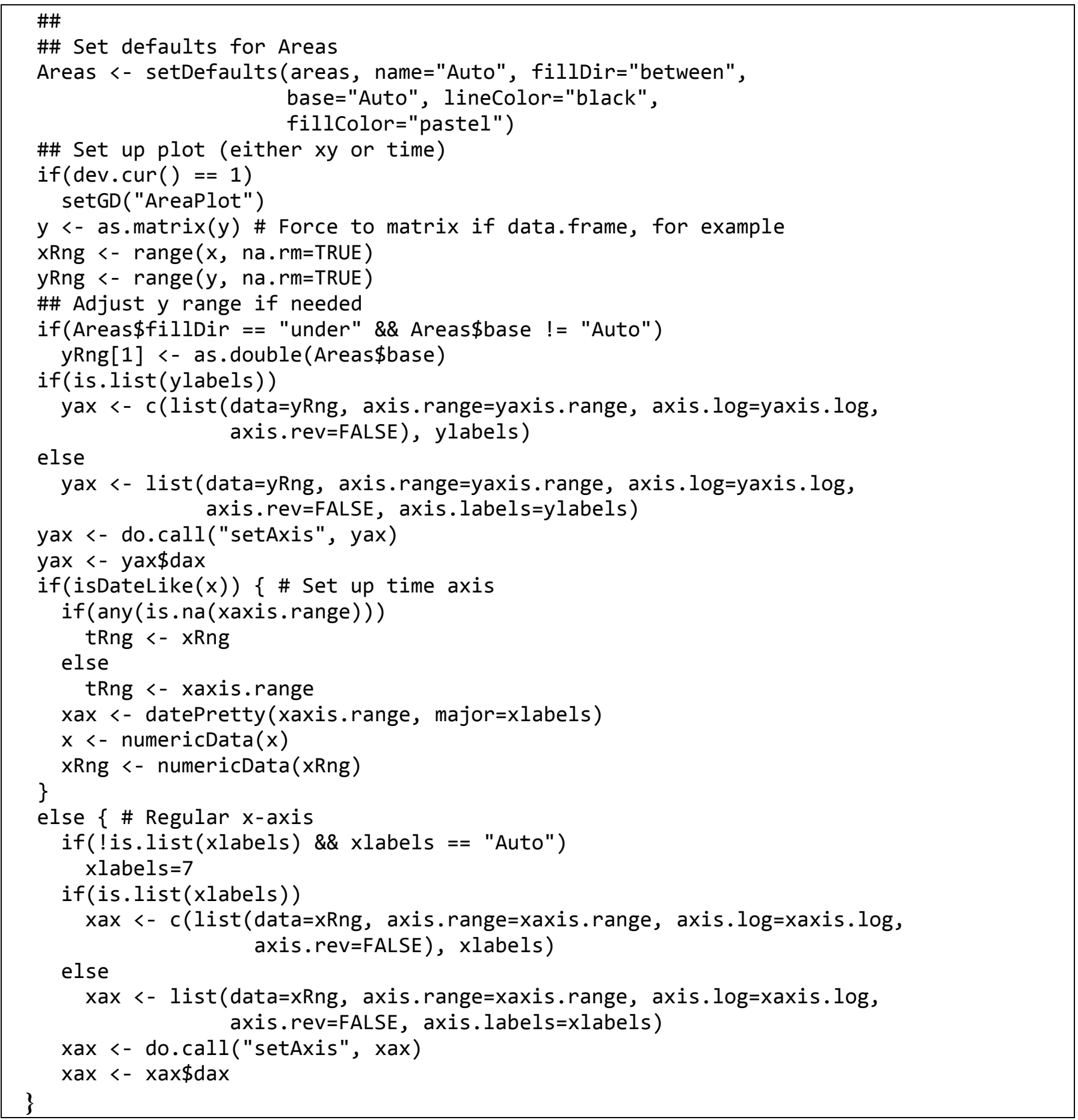


The setMargin function, as used in the following boxed code, replaces the NA values in the margin argument with values derived for the $y$-axis labeling. The $\mathrm{x}$-axis labels are set to defaults that allow for typical labels and the graph title; see the boxPlot function (appendix 6) for ways to set up a wider x-axis margin, for example to accommodate rotated labels. The right-hand margin is set to a small value; thus, to allow for labels, the user must specify a value in margin in the call to areaPlot or other main plotting function. The remaining lines (except for the call to par) simply set up convenience arguments required later in the function.

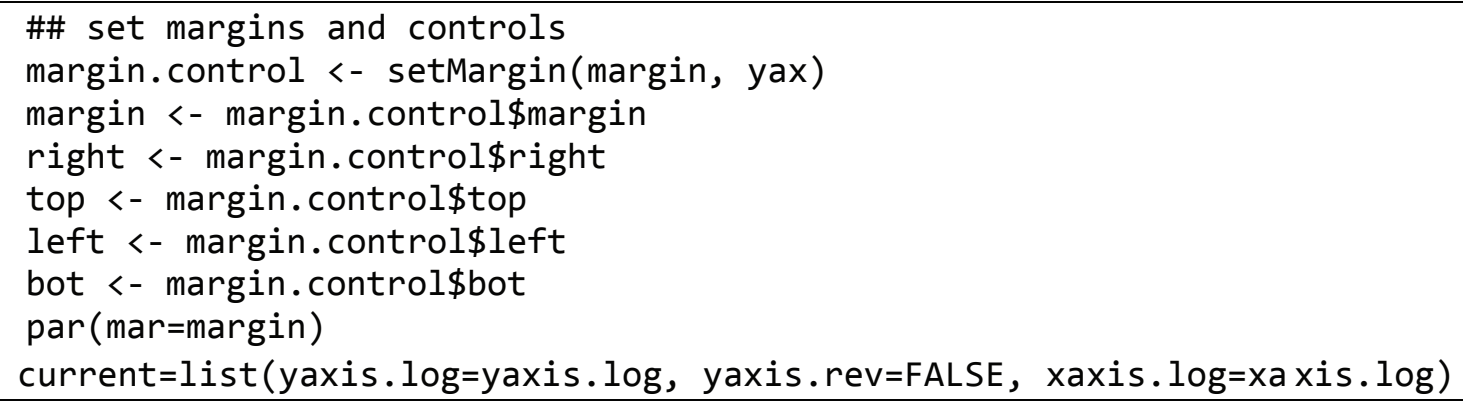

The call to plot in the following boxed code is needed to set up the graph. This call sets up the plotting environment for the subsequent plotting calls, but nothing is actually drawn on the graph. Nothing being plotted is very typical in the main plotting functions in smwrGraphs as it permits finer control of the graphical parameters, and thus, of what will be plotted.

\#\#

plot (xRng, yRng, type='n', xlim=xax\$range, $x a x s=' i '$, axes=FALSE, ylim=yax\$range, yaxs='i', ylab="", $x l a b=" ")$ 
The following boxed code actually draws the plot and is specific to the plotting function, and the general plotting structure will differ from plot type to plot type. The simplest example can be seen in the methods for the $\boldsymbol{x y P l o t}$ function (appendix 7). Other main plotting functions may use base R functions to do the primary computations, but the plotting is done using the specific graphical parameters set up in smwrGraphs; see the histGram function (appendix 9) for an example of such a function. Examining code from a function that is similar to the desired new graph is a good way to proceed. The key to consistent line weights and symbology is to use the contents from the setPlot or setDefaults functions.

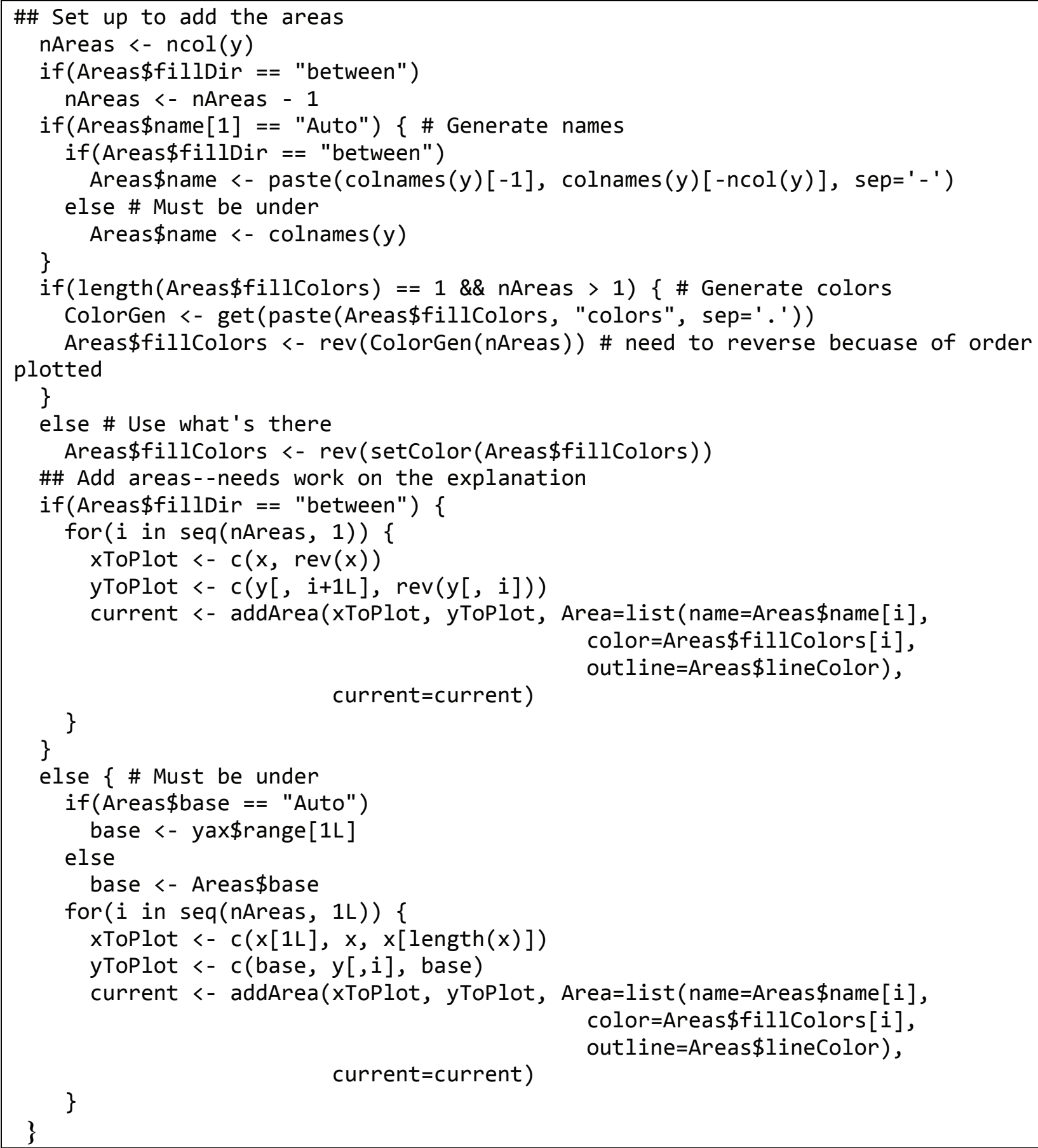


The following remaining boxed code simply finishes the graph, by drawing the bounding box, drawing ticks and labeling the axis, and setting up the object to return. The returned object must contain the axis information and information for the explanation. For areaPlot, the explanation information is added in the calls to addArea.

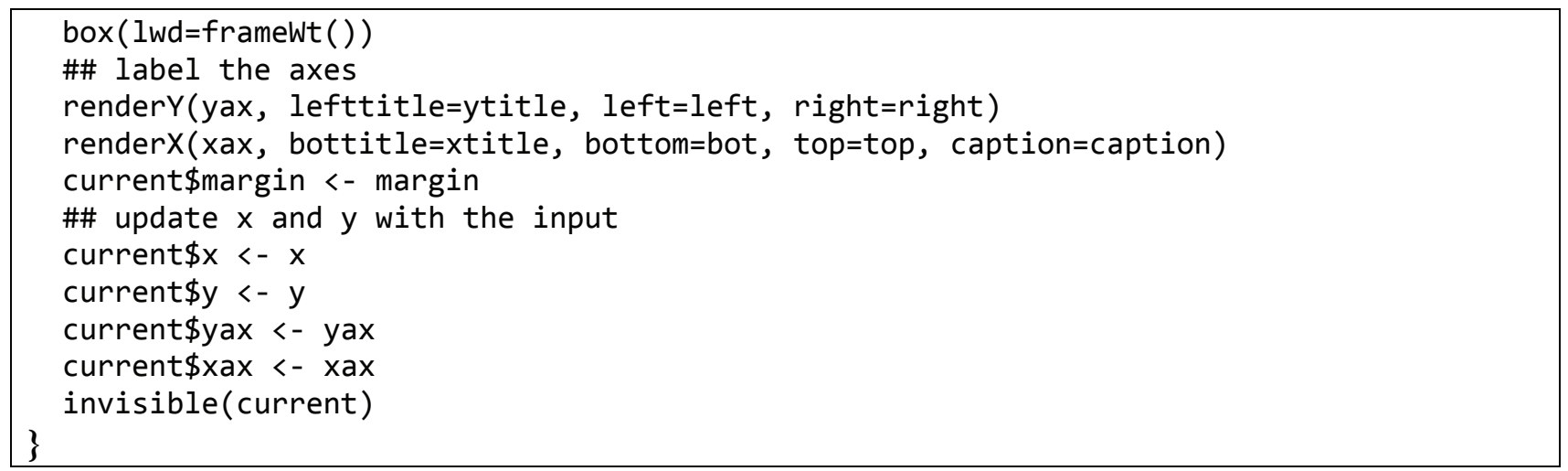

\section{Function to Add to a Graph}

The addArea function is used as an example in this section for adding a graph. The addArea function does not have methods for specific arguments, but the extension to include different methods is straightforward and explained in many books and documentation for R. The example code is presented below in boxed code with comments.

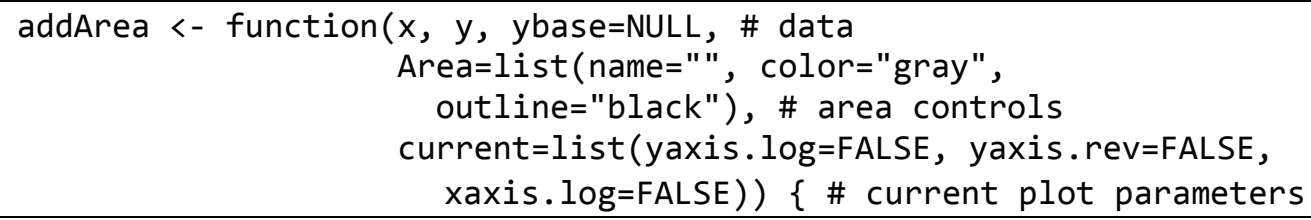

In general, the list of arguments to functions that add to a graph are much shorter than the main plotting functions that create graphs. The first set of arguments in the preceding code box are the data to plot: $x, y$, and $y$ base. The next argument in this example is Area, but the argument Plot commonly is used; both of these arguments describe how the data are to be plotted. The last argument, current, typically contains the output from the main plotting function, but accepts the defaults if no axis was transformed and no explanation will be added.

The following boxed code sets up the data to plot. The transData or numericData function typically is used in this section to transform the data to match the axis transformations of the graph. For this example, the additional code forces the values in ybase to match the other data.

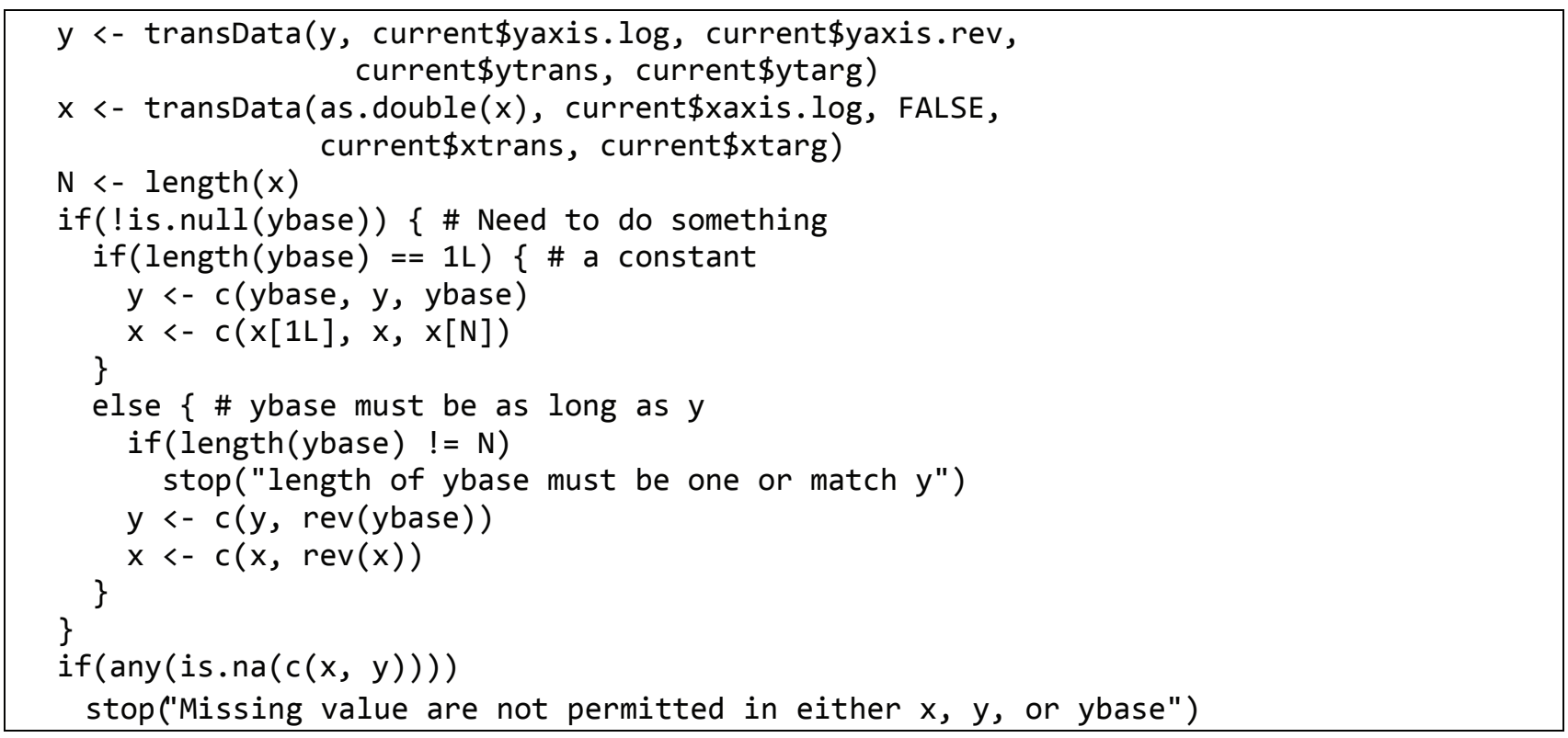


The following boxed code sets the defaults for the Area argument, and demonstrates the setPlot and setExplanation functions, which are required to maintain the explanation information from each plot. For this example, the data are plotted using the polygon function.

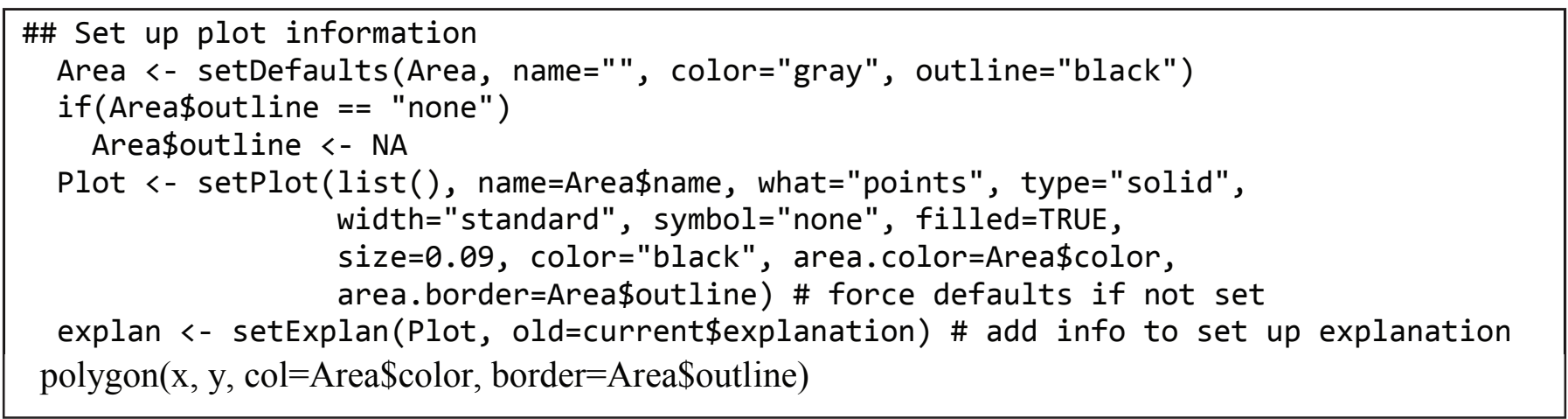

The final following boxed code simply updates the data that were plotted, updates the explanation, and returns that list. Updating the data and explanation is critical for subsequent functions that rely on that information.

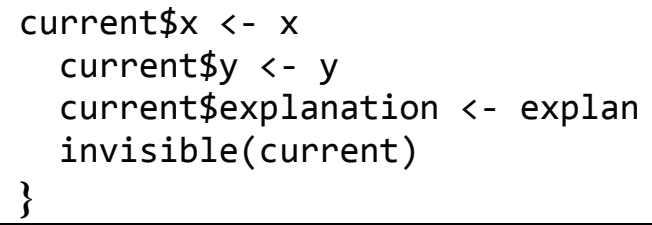

\section{Summary}

This report describes an R packaged called smwrGraphs, which consists of a collection of graphing functions for hydrologic data within $\mathrm{R}$, a programming language and software environment for statistical computing. The functions in the package have been developed by the U.S. Geological Survey to create high-quality graphs for publication or presentation of hydrologic data that meet U.S. Geological Survey graphics guidelines.

In addition to describing the functions that are within smwrGraphs, a Programmer's Guide section that is intended to help programmers contribute new functions to the package is included. The Programmer's Guide section breaks down one main plotting function and one function that adds to a graph into sections and describes the general approach and specific low-level functions used in that section.

\section{Disclaimer}

This package was written by U.S. Federal government employees in the course of their employment and is therefore in the public domain, which means it is not copyrighted and use is unlimited; however, some of the functions depend on other R-packages, which, although free and open source, have more restrictive licensing. Those packages are digest and lubridate [GNU (Gnu's Not Unix) GPL (General Public License), memoise (Massachusetts Institute of Technology,
MIT), XML (Berkeley Software Distribution, BSD). R itself is released under the free software license GNU GPL, either Version 2, June 1991, or Version 3, June 2007. Additional information on licensing is available at https://www.r-project. org/Licenses/ and https://www.gnu.org/licenses/license-list. html\#SoftwareLicenses.

Although this software package has been used by the U.S. Geological Survey (USGS), no warranty, expressed or implied, is made by the USGS or the U.S. Government as to the accuracy and functioning of the program and related program material, nor shall the fact of distribution constitute any such warranty, and no responsibility is assumed by the USGS in connection therewith. This software and related material (functions and documentation) are made available by the USGS to be used in the public interest and the advancement of science. Users may, without any fee or cost, use, copy, modify, or distribute this software, and any derivative works thereof, and its supporting documentation, subject to the USGS Software User's Rights Notice, https://water.usgs.gov/software/ help/notice/.

\section{Acknowledgments}

The authors thanks the time and effort of the U.S. Geological Survey personnel who have used and reviewed this package and contributed suggestions to improve the package. The reviewers were Jared Trost, Minnesota Water Science Center; and Laura De Cicco, Center for Integrated Data 
Analytics. The publication personnel Rebecca Inman, Katherine Laub, Sue Roberts, and Caryl Wipperfurth contributed suggestions and feedback to make the output from the setPDF function quick and easy for USGS illustrators to prepare graphs for USGS publication.

\section{References Cited}

Helsel, D.R., and Hirsch, R.M., 2002, Statistical methods in water resources: U.S. Geological Survey Techniques of Water Resources Investigations, book 4, chapter A3, 522 pages. (Also available at http://pubs.usgs.gov/twri/ twri4a3/).

Lorenz, D.L., 2015, smwrBase-An R package for managing hydrologic data, version 1.1.1: U.S. Geological Survey Open-File Report 2015-1202, 7 p. [Also available at http:// dx.doi.org/10.3133/ofr20151202.]

Lorenz, D.L., Ahearn, E.A., Carter, J.M., Cohn, T.A., Danchuk, W.J., Frey, J.W., Helsel, D.R., Lee, K.E., Leeth, D.C., Martin, J.D., McGuire, V.L., Neitzert, K.M., Robertson, D.M., Slack, J.R., Starn, J., Vecchia, A.V., Wilkison, D.H., and Williamson, J.E., 2011, USGS library for S-PLUS for Windows-Release 4.0: U.S. Geological Survey Open-File Report 2011-1130. [Also available at http://pubs.er.usgs. gov/publication/ofr20111130.]
Piper, A.M., 1944, A graphic procedure in the geochemical interpretation of water analyses: American Geophysical Union Transactions, v. 25, p. 914-923.

R Development Core Team, 2011, R Installation and Administration, Version 2.14.1, 2011-12-22: accessed January 13, 2012, at http://streaming.stat.iastate.edu/CRAN/doc/ manuals/R-admin.pdf.

Stiff, H.A., Jr., 1951, The interpretation of chemical water analysis by means of patterns: Journal of Petroleum Technology, v. 3 , no. 10 , p. 15-17.

Venables, W.N., Smith, D.M., and the R Development Core Team, 2011, An Introduction to R, Version 2.13.1, 2011-0708: accessed August 11, 2011, at http://cran.r-project.org/ doc/manuals/R-intro.pdf. 
Appendixes 


\section{Appendix 1. R Documentation}

The $\mathrm{R}$ documentation is available as a Portable Document Format (PDF) file and can be accessed at https://oi.org/10.3133/ ofr20161188.

\section{Appendix 2. Graph Setup Vignette}

Vignettes are the established R community method for providing examples of how to use the package. The vignette for graph setup is available as a Portable Document Format (PDF) file that describes setting up the graphics environment for smwrGraphs functions and can be accessed at https://doi.org/10.3133/ofr20161188. Examples covered in this vignette include simple graphs, plot customization, axis customization, and multiple graphs.

\section{Appendix 3. Graph Additions Vignette}

Vignettes are the established R community method for providing examples of how to use the package. The vignette for graph additions is available as a Portable Document Format (PDF) file that describes adding plots and other features to graphs created by smwrGraphs functions and can be accessed at https://doi.org/10.3133/ofr20161188. Examples covered in this vignette include reference lines with annotation, grid, smoothed, and regression lines.

\section{Appendix 4. Date Axis Formats Vignette}

Vignettes are the established R community method for providing examples of how to use the package. The vignette for date axis formats is available as a Portable Document Format (PDF) file that describes setting up date and time axis formats for smwrGraphs graphs and can be accessed at https://doi.org/10.3133/ofr20161188. Examples covered in this vignette include hour, day, month, year, and water year formats.

\section{Appendix 5. Graph Gallery Vignette}

Vignettes are the established R community method for providing examples of how to use the package. The vignette for the graph gallery is available as a Portable Document Format (PDF) file that describes miscellaneous smwrGraphs graphs not covered in other vignettes, such as Stiff diagrams (Stiff, 1951), and can be accessed at https://doi.org/10.3133/ofr20161188. Examples covered in this vignette include biplots, scaleless graphs, area graphs, contour plots, correlograms, surface plots, and dendograms.

\section{Appendix 6. Boxplot Vignette}

Vignettes are the established R community method for providing examples of how to use the package. The vignette boxplots is available as a Portable Document Format (PDF) file that describes how to create boxplots using smwrGraphs functions and can be accessed at https://doi.org/10.3133/ofr20161188. Examples in this vignette include various boxplot types.

\section{Appendix 7. Line and Scatter Vignette}

Vignettes are the established R community method for providing examples of how to use the package. The vignette for line and scatter plots is available as a Portable Document Format (PDF) file that describes how to create line and scatter plots using smwrGraphs functions and can be accessed at https://doi.org/10.3133/ofr20161188. Examples in this vignette include scatter, date/time, season, series, color, dot, and scaled plots. 


\section{Appendix 8. Piper Plot Vignette}

Vignettes are the established $\mathrm{R}$ community method for providing examples of how to use the package. The vignette for Piper plots (Piper, 1944) is available as a Portable Document Format (PDF) file that describes how to create Piper plots using smwrGraphs functions and can be accessed at https://doi.org/10.3133/ofr20161188. Examples in this vignette include Piper plots, ternary diagrams, and Stiff diagrams.

\section{Appendix 9. Probability Plots Vignette}

Vignettes are the established R community method for providing examples of how to use the package. The vignette for probability plots is available as a Portable Document Format (PDF) file that describes how to create probability plots using smwrGraphs functions and can be accessed at https://doi.org/10.3133/ofr20161188. Examples in this vignette include probability plots, Q-normal plots, Q-Q plots, and histograms.

Publishing support provided by:

Rolla and Pembroke Publishing Service Centers

For more information concerning this publication, contact: Director, USGS Minnesota Water Science Center 2280 Woodale Drive

Mounds View, Minnesota 55112

(763) 783-3100

Or visit the Minnesota Water Science Center website at: https://mn.water.usgs.gov/ 


\section{$\frac{1}{4}$}

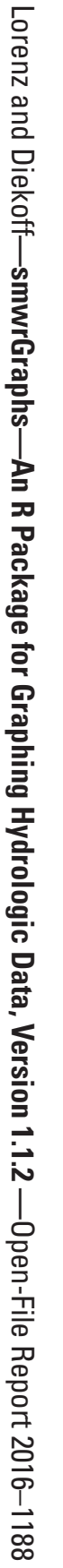

